\title{
Formation of non-thermal particle spectra in galaxy clusters
}

\author{
D.O. Chernyshov ${ }^{* 1}$, V.A. Dogiel ${ }^{1}$, K.-S. Cheng ${ }^{2}$, and C.-M. Ko ${ }^{3}$ \\ ${ }^{1}$ I.E.Tamm Theoretical Physics Division of P.N.Lebedev Institute of Physics, Leninskii pr, 53, \\ 119991 Moscow, Russia \\ ${ }^{2}$ Department of Physics, University of Hong Kong, Pokfulam Road, Hong Kong, China \\ ${ }^{3}$ Institute of Astronomy, National Central University, Jhong-Li 320, Taiwan \\ E-mail: chernyshov@dgap.mipt.ru
}

\begin{abstract}
The origin of hard X-ray (HXR) excess emission from clusters of galaxies is still an enigma, whose nature is debated. One of the possible mechanism to produce this emission is the bremsstrahlung model. However, previous numerical calculations showed that the intracluster plasma should be overheated very fast if the HXR excess is of non-thermal bremsstrahlung origin. We revisited this problem and found that at some parameters of particle acceleration the rate of plasma heating is rather low. For example, for Coma cluster the temperature rise from the initial temperature $T_{0}=7 \mathrm{keV}$ to $T=8 \mathrm{keV}$ takes relatively long time, $\sim 10^{9}$ year, if the momentum diffusion coefficient has a cut-off at low momenta and rises rapidly above the cut-off. At the same time the intensity of non thermal tail is high enough to produce the HXR in e.g. Coma. We conclude that at these conditions the bremsstrahlung model does not contradict the origin of HXR from galaxy clusters and we can explain the HXR satisfactory without heating the plasma too fast.
\end{abstract}

8th INTEGRAL Workshop: "The Restless Gamma-ray Universe"

September 27-30 2009

Dublin, Ireland

* Speaker. 


\section{Introduction}

Hard X-ray excesses (HXR) from several clusters of galaxies discovered by Beppo-SAX, RXTE and INTEGRAL $[1,2,3]$ and references therein) are still an enigma, whose nature is debated. It was assumed in several papers (see e.g. $[4,5]$ and others) that the HXR excess could be due to non-thermal or supra-thermal bremsstrahlung. The problem of this interpretation was discussed by [6] who concluded from simple estimates of the rates of bremsstrahlung and Coulomb energy losses that the intracluster plasma should be overheated very fast if the HXR excess is of non-thermal bremsstrahlung origin. Later on, these conclusions were confirmed by numerical calculations (see $[7,8]$ ).

Below we analyze the processes of plasma heating in conditions of in-situ acceleration in the intracluster medium in order to understand whether processes of plasma overheating exclude indeed the bremsstrahlung interpretation of the HXR from clusters. We start from the analysis of linear model of particle acceleration from the thermal pool.

\section{Particle Acceleration from Background Plasma. Linear Approximation}

This case was analysed first in [9]. He used the kinetic equation which included Coulomb collisions (losses + diffusion) and the Fermi stochastic acceleration, in the form

$$
\frac{\partial f}{\partial t}+\frac{1}{p^{2}} \frac{\partial}{\partial p} p^{2}\left[\left(\frac{d p}{d t}\right)_{C} f-\left\{D_{C}(p)+D_{F}(p)\right\} \frac{\partial f}{\partial p}\right]=0
$$

where $(d p / d t)_{C}$ and $D_{C}(p)$ describe particle energy losses and diffusion in the momentum space due to Coulomb collisions, and $D_{F}(p)$ is the diffusion coefficient of the stochastic Fermi acceleration. It is convenient to introduce dimensionless variables: $\tilde{p}=p / \sqrt{m k T}$, is the dimensionless momentum, $\tilde{t}=t v$, is the dimensionless time, and $\alpha(\tilde{p})=D_{F}(p) /(v m k T)$, is the momentum diffusion coefficient, where $\mathrm{T}$ is the temperature and $v$ is the frequency of thermal particle collisions. It was shown in [9] that the flux of particles running-away into the acceleration energy range for a weak Fermi acceleration (acceleration time $>>$ characteristic time of thermal particle collisions) in the form e.g. $\alpha(\tilde{p})=\alpha_{0} \tilde{p}\left(\alpha_{0}<<1\right)$ was described as

$$
\frac{\partial N}{\partial t}=\sqrt{\frac{2}{\pi}} n v \exp \left(-\frac{\pi}{4 \sqrt{\alpha_{0}}}\right)
$$

where $N$ is the number density of particles running-away into acceleration region, and $n$ is the density of background particles.

Later, it was shown in $[4,10]$ that such an acceleration could provide a flux of suprathermal electrons necessary for the HXR excess in Coma.

A disadvantage of this approximation is that the temperature of background plasma is supposed to be constant in accordance with the linear approximation, and the sole evolution of a non-thermal "tail" can be analysed. Analysis of plasma heating by accelerated particles can be performed in the framework of non-linear models. 


\section{Particle Acceleration from Background Plasma. Non-Linear Approximation}

The non-linear case was analysed in [5] who showed that the stochastic acceleration after $\sim$ $10^{9}$ years formed the electron distribution with the temperature about $8 \mathrm{keV}$ and a tail of nonthermal particles with the intensity needed to explain the Beppo-SAX data. Later on [7] derived the more general kinetic equation. The equation has the form

$$
\frac{\partial f(\mathbf{u})}{\partial t}=\frac{\partial}{\partial u_{\alpha}}\left[\left(D_{\alpha \beta}+D_{\alpha \beta}^{F}\right) \frac{\partial f(\mathbf{u})}{\partial u_{\beta}}-F_{\alpha} f(\mathbf{u})\right]
$$

where $\mathbf{u}$ is the ratio of particle momentum to its rest mass. The coefficients $D_{\alpha \beta}$ and $F_{\alpha}$ are determined by Coulomb collisions of the particles:

$$
D_{\alpha \beta}=A \int Z_{\alpha \beta}\left(\mathbf{u}, \mathbf{u}^{\prime}\right) f\left(\mathbf{u}^{\prime}\right) d^{3} u^{\prime}, \quad F_{\alpha}=-A \int\left[\frac{\partial}{\partial u_{\beta}^{\prime}} Z_{\alpha \beta}\left(\mathbf{u}, \mathbf{u}^{\prime}\right)\right] f\left(\mathbf{u}^{\prime}\right) d^{3} u^{\prime}
$$

where

$$
\begin{aligned}
& Z_{\alpha \beta}\left(\mathbf{u}, \mathbf{u}^{\prime}\right)=\frac{r^{2}}{\gamma \gamma^{\prime} w^{3}}\left[w^{2} \delta_{\alpha \beta}-u_{\alpha} u_{\beta}-u_{\alpha}^{\prime} u_{\beta}^{\prime}+r\left(u_{\alpha} u_{\beta}^{\prime}+u_{\alpha}^{\prime} u_{\beta}\right)\right] \\
& A=\frac{8 \pi n e^{2}\left(e^{\prime}\right)^{2}}{m^{2}} \ln \Lambda, \quad r=\gamma \gamma^{\prime}-\mathbf{u} \cdot \mathbf{u}^{\prime} / c^{2}, \quad w=c \sqrt{r^{2}-1}, \quad \gamma=\sqrt{1+\mathbf{u}^{2} / c^{2}}
\end{aligned}
$$

The stochastic Fermi acceleration is described by the tensor $D_{\alpha \beta}^{F}$ which is supposed to be isotropic. $D_{\alpha \beta}^{F}=D_{F} \delta_{\alpha \beta}$. With this equation, [7] calculated the spectrum evolution. They took the Fermi diffusion coefficient as a power-law function of the velocity $u$ with a cut-off at $u / c=0.5$, i.e.

$$
D_{F}(u)=K u^{\varsigma} \theta(u-c / 2)
$$

where $K$ is a constant, $\varsigma=5 / 3$ and $\theta(x)$ is the Heaviside function. The characteristic time of acceleration at $u=c / 2$ is about $2 \cdot 10^{8}$ years for the chosen parameters. They showed that after only $\sim 10^{7}$ years (about only $2 \%$ of the time required by [5]) the electron distribution had involved a nonthermal tail that might account for the HXR of the Coma Cluster but after that the background plasma gained too much heat to account for the thermal, soft component that was in conflict with the conclusions of [5].

The following results of [8] validated conclusions from [7], and were even more extreme for the case of in-situ acceleration in Coma if the HRX origin is bremsstrahlung. They took the momentum diffusion coefficient in the form

$$
D_{F}(E)=\frac{E^{2}}{\xi(E) \tau_{0}\left(1+E_{c} / E\right)^{\varsigma}}
$$

where $E$ is the kinetic energy, $E_{c} \simeq 0.25 \mathrm{keV}$, and $\tau_{0}$ is the characteristic time of acceleration. The authors concluded that the continuous stochastic emission of thermal electrons for the case of HXR excess of Coma cannot work because the energy gained by the particles was distributed to the whole plasma on timescales much shorter than that of the acceleration process. At acceleration rates smaller than the thermalization rate of the background plasma, there is very little acceleration. The primary effect is the heating of the plasma. In the opposite case, at higher energizing rates, a 
nonthermal tail is developed with the intensity equaled or even higher than necessary to produce by bremsstrahlung the non-thermal X-ray flux from Coma, but this is again accompanied by an unacceptably high rate of heating. The background temperature is increased to $20 \mathrm{keV}$ from an initial value of $8 \mathrm{keV}$ within 5 million years.

In other words, we can summarize the result of $[7,8]$ as: there are no conditions when the temperature of background plasma is about $8 \mathrm{keV}$ distribution while the intensity of nonthermal tail is high enough for the HRX from Coma if particle are accelerated longer than $10^{5}$ years.

Below we show that the situation for in-situ acceleration is not so despairing, and at some conditions we can satisfy all these requirements of the model, i.e we can find a set of the parameters when an intensive tail of accelerated particles is formed for the time comparable with the lifetime of clusters while the plasma is still relatively cold.

\section{Numerical Calculations}

We investigate the problem of plasma heating and particle acceleration using the following set of parameters. The initial plasma parameters are: the density $n_{0}=10^{-4} \mathrm{~cm}^{-3}$ and the initial temperature $T_{0}=7 \mathrm{keV}$. For calculations we use the nonlinear equations (3.1) with the coefficients presented by Eqs. (3.2)-(3.4). The velocity is $u=p / m$ and the dimensionless time is $\tilde{t}=t / \tau_{\text {Coul }}$ where $\tau_{\text {Coul }}$ is

$$
\tau_{\text {Coul }}=\frac{4 \pi n_{0} e^{4}}{m^{2} c^{3}} \ln \Lambda \sim 10^{16} \mathrm{~s}
$$

The starting temperature in calculations was taken to be $T_{0}=7 \mathrm{keV}$. The momentum diffusion coefficient is taken in the form

$$
\alpha(u)=\alpha_{0} u^{\varsigma} \theta\left(u-u_{0}\right)
$$

The dimensionless parameter of stochastic acceleration is $\alpha_{0}=\tau_{C o u l} / \tau_{F}$ where $\tau_{F}$ is the characteristic time of stochastic acceleration. This set of parameters $\left\{T_{0}, n_{0}, \alpha_{0}, u_{0}, \varsigma\right\}$ determines the spectrum evolution. The equation Eq. (3.1) describes non-stationary situation since there is an external source of energy input in the form of stochastic acceleration but there are no terms in the equation describing an energy sink. Therefore, stationary spectra of electrons are not expected. Our goal is to find a set of parameters of acceleration for Coma at which the plasma is not overheated for long enough time and its temperature does not exceed $8 \mathrm{keV}$ too much, and at the same time the intensity of electrons in the nonthermal region is high enough to produce the observed HXR excess. Below we calculate the temperature variations $T(t)$ as a function of time for different parameters of $\alpha(u)$ in the form (4.2), and present the intensity of non-thermal X-rays for the moment $t$ when the plasma temperature reaches the value $8 \mathrm{keV}$. The results are shown in Fig. 1. One can see that for $u_{0}=0$ the plasma is overheated for a very short time that agrees completely with the conclusion of [8]. Increase of $u_{0}$ leads to a slower heating but the intensity of nonthermal particles is also decreasing with $u_{0}$. We see that for $u_{0}=0.55 \mathrm{c}$ the plasma temperature is almost constant and equals about $8 \mathrm{keV}$ though the intensity of the hard X-ray radiation is quite high.

Variations of the spectral index $\varsigma$ from Eq. 4.2 significantly change the rate of plasma heating, the higher is $\varsigma$ the smaller rate of temperature increase. 

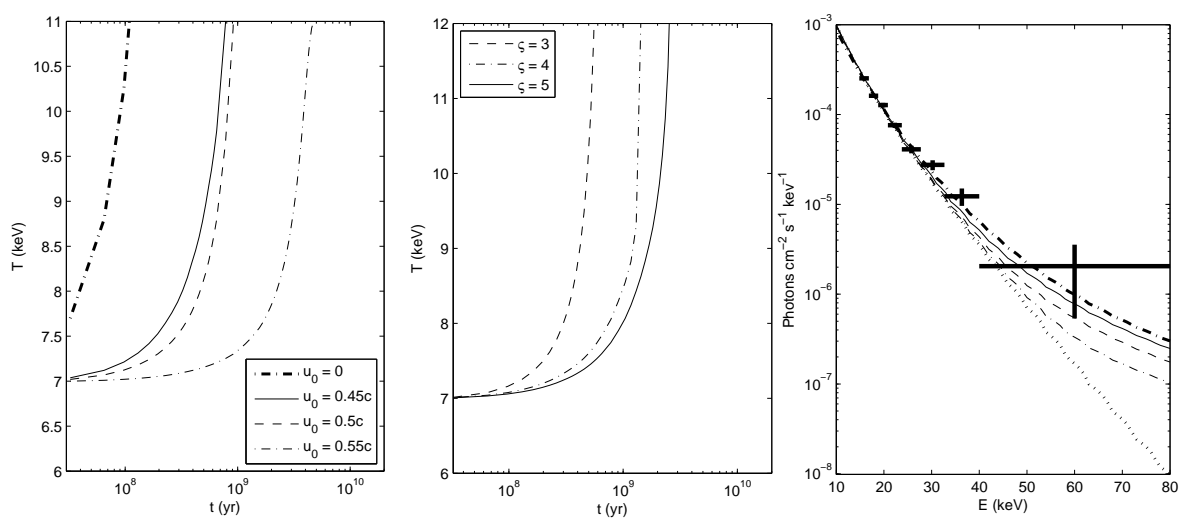

Figure 1: The panel on the left: Variations of the plasma temperature for $\alpha_{0}=1.7 \cdot 10^{-17} \mathrm{~s}^{-1}, \varsigma=5$ as affected value of $u_{0}$ (see Eq.(4.2)). The middle panel: Variations of the plasma temperature for $\alpha_{0}=$ $1.7 \cdot 10^{-17} \mathrm{~s}^{-1}, u_{0}=0.5 c$ as affected value of $\varsigma$. The panel on the right: Intensity of hard X-ray emission from Coma at the moment when $\mathrm{T}=8 \mathrm{keV}$ for $\alpha_{0}=1.7 \cdot 10^{-17} \mathrm{~s}^{-1}, \varsigma=5$ as affected value of $u_{0}$ shown in the left panel. Crosses are the data from [1].

If we assume that $\alpha(u)$ is power-law but becomes constant at high values of $u$ e.g.

$$
\alpha(u)=\alpha_{0} \frac{\left(u / u_{0}\right)^{5}}{1+(u / 5 c)^{5}} \theta\left(u-u_{0}\right)
$$

where $u_{0}=0.55 \mathrm{c}$, then as shown in Fig. 4 the increase of temperature is negligible for the time $t>10^{8} \mathrm{yr}$, and the intensity of hard X-rays is high enough to explain the flux from Coma.
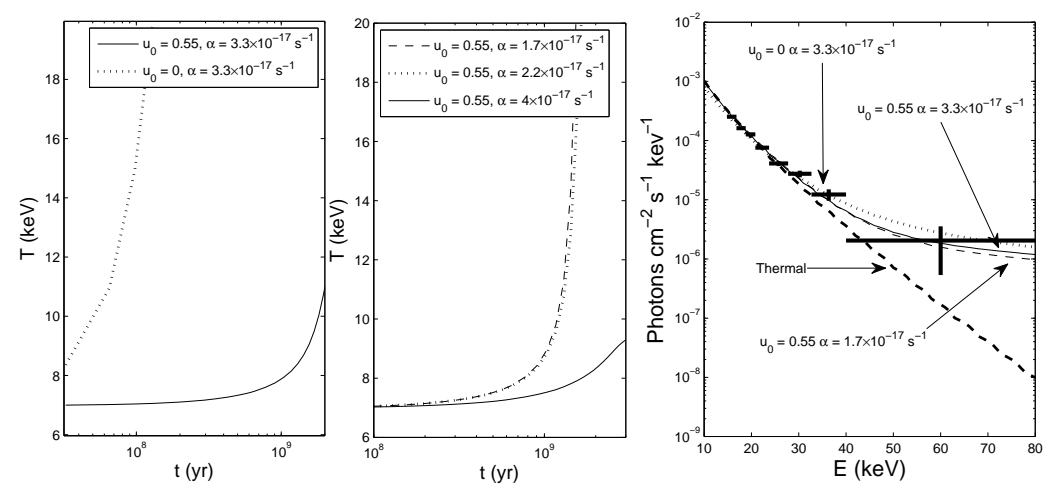

Figure 2: Variations of temperature and the intensity of hard X-ray emission for the momentum diffusion in the form (4.3).

\section{Conclusion}

- If there is no cut-off of the acceleration at small velocities (i.e. $u_{0}=0$ ) the processes of acceleration are similar to that presented in [8]. The plasma is overheated (or all background electrons are accelerated) for a very short time $\sim 10^{5}-10^{6}$ years; 
- If $\alpha(u)=\alpha_{0} u^{5 / 3} \theta(u-c / 2)$, the plasma is overheated for the time $\sim 10^{6}-10^{7}$ years just as in [7];

- For sufficiently large values of $\varsigma \sim 5$ the rate of plasma heating is rather low. The temperature increase from the initial temperature $T_{0}=7 \mathrm{keV}$ to $T=8 \mathrm{keV}$ takes relatively long time $\geq 3 \times 10^{8}$ years. At the same time the intensity of non thermal tail is high enough to produce the HXR in Coma;

- The bremsstrahlung model is able to explain the HXR from galaxy clusters without heating the plasma too fast and that is consistent with the observed plasma temperature.

\section{Acknowledgments}

VAD and DOC are partly supported by the NSC-RFBR Joint Research Project RP09N04 and 09-02-92000-HHC-a. KSC is supported by a grant under HKU 7011/10p. CMK is supported in part by the National Science Council, Taiwan, under grants NSC 98-2923-M-008-001-MY3 and NSC 99-2112-M-008-015-MY3. The authors are very grateful to the unknown referee fro the text corrections.

\section{References}

[1] Fusco-Femiano, R., Landi,R. \& Orlandini M., Nonthermal Hard X-Ray Excess in the Coma Cluster: Resolving the Discrepancy between the Results of Different PDS Data Analyses, ApJL 654 (2007) L9

[2] Rephaeli, Y., Nevalainen, J., Ohashi, T. \& Bykov A. M., Nonthermal Phenomena in Clusters of Galaxies, Space Science Reviews 134 (2008) 71

[3] Eckert, D., Produit, N., Paltani, S. et al., INTEGRAL discovery of non-thermal hard X-ray emission from the Ophiuchus cluster, A\&A 479 (2008) 27

[4] Dogiel, V. A., On the thermal origin of the hard X-ray emission from the Coma cluster, A\&A 357 (2000) 66

[5] Blasi, P., Stochastic Acceleration and Nonthermal Radiation in Clusters of Galaxies, ApJL 532 (2000) L9

[6] Petrosian, V., On the Nonthermal Emission and Acceleration of Electrons in Coma and Other Clusters of Galaxies, ApJ 557 (2001) 560

[7] Wolfe, B., \& Melia, F., Covariant Kinetic Theory with an Application to the Coma Cluster, ApJ 638 (2006) 125

[8] Petrosian, V. \& East, W.E., Heating and Acceleration of Intracluster Medium Electrons by Turbulence, ApJ 682 (2008) 175

[9] Gurevich, A. V., Amount of Accelerated Particles in an Ionized Gaz, Sov. Phys. JETP 38 (1960) 1150

[10] Dogiel, V. A., Colafrancesco, S., Ko, C.-M. et al., In-situ acceleration of subrelativistic electrons in the Coma halo and the halo's influence on the Sunyaev-Zeldovich effect, A\&A, 461 (2007) 433 